\title{
A Cell-Based Light Interaction Model for Human Blood
}

\author{
D. Yim, G.V.G. Baranoski, B.W. Kimmel, T.F. Chen \& E. Miranda
}

Natural Phenomena Simulation Group, D.R. Cheriton School of Computer Science, University of Waterloo, Canada

\begin{abstract}
The development of predictive appearance models for organic tissues is a challenging task due to the inherent complexity of these materials. In this paper, we closely examine the biophysical processes responsible for the appearance attributes of whole blood, one the most fundamental of these materials. We describe a new appearance model that simulates the mechanisms of light propagation and absorption within the cellular and fluid portions of this specialized tissue. The proposed model employs a comprehensive, and yet flexible first principles approach based on the morphological, optical and biochemical properties of blood cells. This approach allows for environment driven changes in the cells' anatomy and orientation to be appropriately included into the light transport simulations. The correctness and predictive capabilities of the proposed model are quantitatively and qualitatively evaluated through comparisons of modeled results with actual measured data and experimental observations reported in the scientific literature. Its incorporation into rendering systems is illustrated through images of blood samples depicting appearance variations controlled by physiologically meaningful parameters. Besides the contributions to the modeling of material appearance, the research presented in this paper is also expected to have applications in a wide range of biomedical areas, from optical diagnostics to the visualization and noninvasive imaging of blood-perfused tissues.
\end{abstract}

Categories and Subject Descriptors (according to ACM CCS): I.3.7 [Computer Graphics]: Three-Dimensional Graphics and Realism I.3.8 [Computer Graphics]: Applications

\section{Introduction}

The realism of computer generated images is directly associated with the careful modeling of material appearance, i.e., how different materials reflect, transmit and absorb light. Accordingly, one of the focal points of computer graphics research has been the development of light interaction models to enable the rendering of believable images of a wide range of man-made and natural materials. In recent years, substantial efforts have been directed toward the development of models that can also be used in a predictive manner, extending their scope of applications to other disciplines. For example, predictive models of light interaction with organic materials can be employed in the design of more effective diagnostic procedures and instruments [LÖ93, FRMM06, BK10]. However, despite the remarkable progress that has been achieved in material appearance modeling [DRS08], predictive solutions for fundamental organic materials remain elusive. In this paper, we investigate the interactions of light with one of the most important of these materials, human blood, a highly specialized type of con- nective tissue in which cells are suspended and carried in a fluid called plasma.

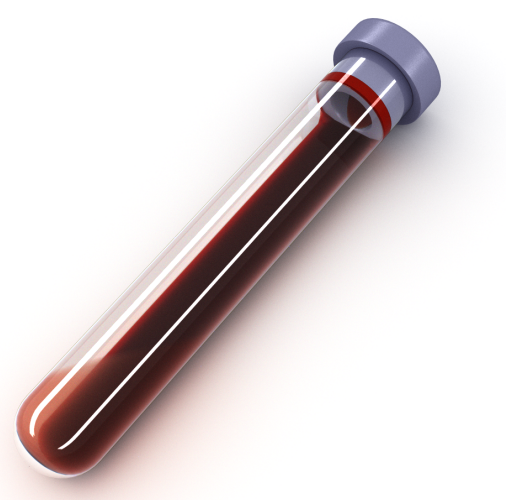

Figure 1: Image rendered using the proposed model of light interaction with human blood. 
The spectral and spatial distributions of light incident on whole blood not only determine its appearance attributes (e.g., color and translucency), but also provide crucial information about the medical condition of an individual. For example, the noninvasive optical monitoring of oxygen saturation, known as pulse oximetry, is arguably one of the most important methods for assessing the well-being and safety of patients during surgery, anesthesia recovery and critical care [Fle95, FRMM06]. Computations of light propagation in blood are also often performed to correlate theoretical results to measured values in order to determine changes to biochemical and morphological characteristics of blood triggered by pathological conditions [RFD*99, FRMM06, OS11, YBK*11]. Furthermore, the appearance of blood-perfused tissues are significantly affected by tissue blood content since the properties of whole blood itself are substantially different from those of soft tissues [YPR ${ }^{*}$ 02]. For these reasons, predictive simulations of light interactions with blood controlled by physiologically meaningful parameters are also of great importance for the imaging, screening, diagnosis and treatment of tissue disorders [LÖ93, SL98, BK10].

In this paper, we present a novel model for the predictive simulation of light interactions with blood. The proposed model employs a first principles approach that takes into account the specific morphological and optical properties of blood cells, to compute both the spectral (reflectance and transmittance) and spatial (scattering) responses associated to the appearance attributes of blood. Using this approach, the proposed cell-based model of light interaction with whole blood, herein referred to as the CLBlood model, can account for changes in the biophysical properties of blood cells and plasma under different physiological conditions and rheological states (stationary and steady flow). We remark, however, that our investigation focuses on lightmatter interaction issues, i.e., the modeling of blood flow dynamics is beyond the scope of this work. Since our research is aimed not only at rendering applications, but also at biomedical applications, the CLBlood model is designed to provide multispectral predictions in the ultraviolet (UV), visible and near-infrared (NIR) regions of the light spectrum. The correctness of these predictions are quantitatively and qualitatively evaluated through detailed comparisons with actual measured data and observations of blood optical phenomena reported in the scientific literature. We also present images rendered using the CLBlood model to further illustrate its predictive capabilities and its use in realistic image synthesis.

\section{Related Work}

In the biomedical field, models based on the Twersky's theory [AS67] and the diffusion theory [SS88] have been employed in the estimation of blood optical parameters. While the original Twersky's theory do not describe the spatial dis- tribution of the reflected and transmitted light as reported by Steinke and Shepherd [SS88], the original diffusion theory (DT) provide valid approximations for light transport in a medium whose absorption coefficient is significantly smaller than its scattering coefficient as stated by Sardar and Levy [SL98] and Yaroslavsky et al. [YPR*02]. Since this condition is not satisfied for blood in visible spectral domain [SL98, YPR* 02], blood optics researchers usually combine DT with other analytical approaches to overcome this limitation (e.g., [SSH*08]), or resort to numerical methods as described in a comprehensive blood optics review by Yaroslavsky et al. [YPR*02]. Accordingly, numerical adding-doubling [SL98] and Monte Carlo methods [YYGS99, RFD*99, FRMM06] have been also applied in inverse algorithms for the determination of blood optical properties and in two-dimensional simulations aimed at the NIR detection of blood coagulation [OS11]. In general, the accuracy of simulations based on these analytical and numerical approaches is limited by oversimplifications with respect to the absorption and scattering properties of blood components. For example, light is often assumed to traverse a blood cell without any internal reflections, which can result in significant deviations from the actual absorption profile of whole blood [KESJ51, RFD*99]. Also, the collective scattering behavior of whole blood is often simulated using empirical phase functions or scattering theories that represent blood cells by cylinders, spheres or ellipsoids, and these approximations can lead to significant deviations from the actual scattering profile of whole blood [FRMM06, KKKM11].

Few computer graphics related papers addressing the appearance of blood have been published to date. In visualization works aimed at applications in biology and medicine (e.g., [HTP* 08, PH07]), the appearance of blood is usually rendered using simplified color coded schemes. In realistic rendering applications, models of light interaction with human tissues (e.g., [DJ06, BK10]) account for the presence of blood through the incorporation of absorption coefficients of blood-borne pigments in the simulations. Although there are a number of detailed models aimed at the realistic rendering of fluid media (e.g., [FCJ07, GSAA08]) and particulate materials (e.g., [KB07, $\left.\left.\mathrm{JAM}^{*} 10\right]\right)$, these models are not designed to account for specific morphological, optical and physiological factors affecting light interactions with blood cells. These factors are essential for the predictive modeling of whole blood appearance. Furthermore, to the best of our knowledge, none of the existing material appearance models accounts for rheological effects on the specific optical properties of material constituents. These effects can result in noticeable changes in the absorption and scattering profiles of whole blood [Bit86,LÖ93].

\section{Blood Optical Properties}

The cellular portion of blood is called formed elements, and it includes the erythrocytes or red blood cells (RBCs), the 
white blood cells (WBCs) and the platelets (PLTs) [dG95]. Although the volume fraction of human blood occupied by the formed elements, clinically known as the hematocrit and denoted by $H C T$, usually varies from $0.37-0.52$, lower values can also be observed under in vivo conditions (e.g., during heart surgery, the patient's hematocrit is usually lowered $\left.\left[\mathrm{MGF}^{*} 05\right]\right)$, and may be lower than 0.01 under in vitro conditions (e.g., during blood optics investigations and disease screening tests [YPR ${ }^{*}$ 02,FRMM06]). The optical properties of human blood under normal physiological conditions are largely determined by light interactions with plasma and RBCs [YPR* 02], which account for $99 \%$ of the formed elements [MMFH07]. The effects of the optical properties of WBCs and PLTs on the light scattering and absorption by whole blood are considered negligible [YPR $\left.{ }^{*} 02, \mathrm{MGF}^{*} 05\right]$.

RBCs have a thin plasma membrane that encloses mainly a hemoglobin solution. The absorption and scattering of light by the RBCs are two to three orders of magnitude higher than those of the other blood components [MMFH07]. The light scattered by a single RBC depends on its shape, volume, refractive index and orientation [KKKM11]. Normal RBCs are characterized by a biconcave disk shape (Fig 2), with a volume, surface area and diameter ranging from $80-108 \mu m^{3}, 119-151 \mu m^{2}$ and $7.2-8.44 \mu m$ respectively [YPR $\left.{ }^{*} 02\right]$. Their refractive index corresponds essentially to the refractive index of the hemoglobin solution due to the negligible thickness $(\approx 7 \mathrm{~nm})$ of their membrane [TMEG85]. With respect to the angular distribution of the scattered light, the RBCs are characterized by a strong forward scattering behavior, with an average scattering angle about $5^{\circ}$ [Lat84] and an exponential fall-off [KKKM11].

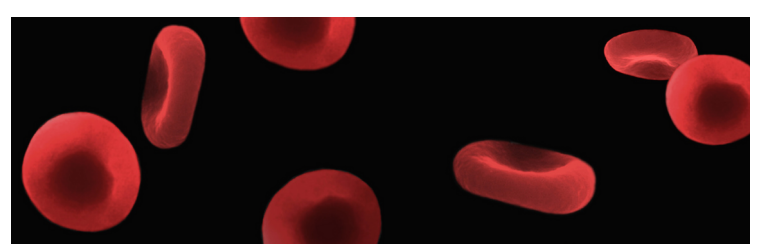

Figure 2: Color coded scanning electron micrograph of red blood cells (Credits: Dennis Kunkel Microscopy, Inc./Visuals Unlimited/Corbis).

The absorption of light by the RBCs is dominated by hemoglobin in its functional, oxygen-binding, forms [Fle95], namely oxyhemoglobin and deoxyhemoglobin. Other types of hemoglobin that do not bind oxygen reversibly, known as dysfunctional hemoglobins, namely methemoglobin, carboxyhemoglobin and sulfhemoglobin, comprise less than $3 \%$ of the total hemoglobin content in healthy individuals [Fle95, YPR*02]. Water also plays a minor role in the absorption of light by RBCs, notably in the NIR range. Since RBCs are essentially a package of hemoglobin solution, the Beer-Lambert law can be employed to quantify the absorption of light traveling within this solution [TMEG85]. It is necessary to take into account, however, that the optical path of light traveling within a cell may increase due to multiple reflections at its internal boundary [KESJ51, RFD*99]. Hence, the amount of light absorbed within the RBC depends on the cell shape, orientation with respect to the incident light as well as its hemoglobin concentration and oxygen saturation level, $\mathrm{SaO}_{2}$, which determines the fractions of oxyhemoglobin and deoxyhemoglobin.

Pure plasma consists of approximately $90 \%$ water, 7-9\% proteins (about $60 \%$ albumin, 36\% globulin and $4 \%$ fibrinogen) with the balance being composed of electrolytes, metabolic intermediates, vitamins, nutrients and waste products [dG95]. RBCs may also release hemoglobin into plasma due to the damage of their membrane caused by mechanical or chemical impact, a process known as hemolysis [RFD*99]. Plasma absorption in UV to visible range is associated with the absorption due to chromophores found in proteins and other molecules, while in the NIR range its is dominated by water absorption. The scattering properties of pure plasma are mainly described by Rayleigh scattering of protein molecules [MMFH07]. Although plasma shows weak absorption and scattering behaviors when compared to the RBCs, it has a noticeable influence on the absorption profile of whole blood due to its refractive index differences with the RBCs. These differences may result in an increase of the path length of light traversing the RBCs [RFD*99], which, in turn, increase the probability of light being absorbed within these cells.

The scattering and absorption properties of whole blood are significantly affected by its motion state measured in terms of its shear rate, which is defined as the velocity gradient in the direction normal to the flow [LÖ93]. At low shear rates, the cells are randomly oriented. As shear rate increases, many randomly oriented cells start to exhibit angular rotation (rolling or tumbling) and align with the major axis parallel to the flow direction. Although the degree of alignment increases with the shear rate, it also depends on the hematocrit. At high shear rates and high hematocrit (above 0.4), the alignment becomes more pronounced and the cells start to show some degree of elongation [Bit86, LÖ93].

\section{The CLBlood Model}

The proposed model employs a simulation framework in which light is represented by discrete rays, each one traveling at a given wavelength $\lambda$. Within this framework, the interactions of light with plasma and individual cells are modeled as a random walk process. Scattering and absorption events are probabilistically accounted for using data driven procedures and wave optics resources when appropriate.

The CLBlood simulation pipeline can be divided into four main stages: cell representation, plasma-light interactions, cell positioning and cell-light interactions. 
These stages are implemented using Monte Carlo methods that rely on the generation of random numbers $\xi_{i}$, for $i=1,2, \ldots, 9$, uniformly distributed in the interval $[0,1]$. The following description of this pipeline concentrates in the red blood cells due their dominant optical role. The interactions of light with other cells, such as WBCs and PLTs, can be handled similarly to the RBCs and are implicitly addressed in the model formulation by analogy.

\subsection{Cell Representation}

When a ray hits a cell, it can be reflected back to the surrounding medium or transmitted to the cell interior. In order to compute the probabilities of light reflection and absorption by a cell, we need a geometrical representation that can closely approximate its shape under different conditions. Furthermore, this representation needs to support operations performed during the subsequent simulation stages, namely the calculation of the cell volume and cross-sectional area (in the direction perpendicular to the ray propagation direction), the computation of ray-cell (internal boundary) intersections and the selection of random points on the surface of the cell facing the ray propagation direction.

Accordingly, we created a parametric NURBS representation [MCFS00] for the red blood cells (Figure 3). This representation allows us to accurately perform the required operations and effectively handle changes in the cells' morphology such as elongation and swelling. We used the biconcave cross-section profile of an unstressed red blood cell [SZC73] to adjust the control points of the NURBS representation in order to have its volume and diameter within the typical range of normal RBCs. Standard rejection sampling techniques were then used to calculate the volume and the crosssectional area of our in silico cells [YBK $\left.{ }^{*} 11\right]$.

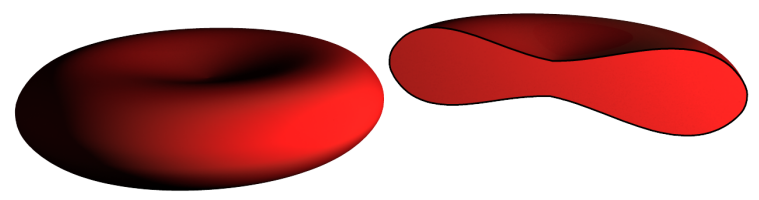

Figure 3: Sketch depicting the NURBS representation of biconcave red blood cells employed by the CLBlood model. Left: whole volume. Right: cross section.

\subsection{Plasma-Light Interactions}

When a ray first interacts with a blood volume, a Fresnel test is performed [BK10], i.e., the Fresnel coefficient, $F$, at the interface between plasma and the surrounding medium is computed, and if $\xi_{1} \leq F$, then the ray is reflected back to the surrounding medium. Otherwise, it is transmitted to the fluid portion of blood where it may travel a distance $d$ before hitting another interface with the surrounding medium or a cell. In both cases, Fresnel tests are performed to determine the next step in the random walk process. The computation of the distance $d$ and the Fresnel tests involving the cells are described in the next sections.

A ray traversing a distance $d$ in the plasma may be subject to scattering and absorption events. To account for scattering caused by protein molecules, we initially compute the Rayleigh scattering coefficient [McC76]:

$$
\mu_{s}(\lambda)=\frac{128 \pi^{5} r^{6}(c / M)}{3 \lambda^{4}}\left(\frac{\left(\eta_{p} / \eta\right)^{2}-1}{\left(\eta_{p} / \eta\right)^{2}+2}\right)^{2}
$$

where $\eta, c, r$, and $M$ respectively represent the refractive index, concentration, molecular radius and weight of each type of protein, and $\eta_{p}$ corresponds to the refractive index of plasma. We then employ $\mu_{s}(\lambda)$ to compute the Rayleigh scattering probability for each type of protein using [BK10]:

$$
P_{\mu_{s}}(\lambda)=1-e^{-\mu_{s}(\lambda) d} .
$$

Finally, if $\xi_{2}<P_{\mu_{s}}(\lambda)$, then the ray direction is perturbed according to the Rayleigh scattering phase function [BK10]. For simplicity, this perturbation is implemented using rejection sampling as shown in Algorithm 1, where $\theta_{R}$ represents the polar perturbation angle, and $\phi_{R}$ corresponds to the azimuthal perturbation angle.

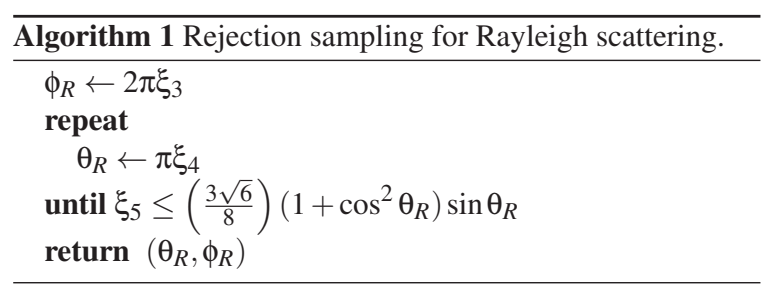

To compute the absorption probability, we employ a stochastic representation of the Beer-Lambert law [BK10]:

$$
P_{\mu_{a_{p}}}(\lambda)=1-e^{-\mu_{a_{p}}(\lambda) d},
$$

where $\mu_{a_{p}}(\lambda)$ corresponds to the effective absorption coefficient of plasma. After $P_{\mu_{a_{p}}}(\lambda)$ is computed, the testing is performed, i.e., if $\xi_{6}<P_{\mu_{a p}}(\lambda)$, then the ray is terminated.

\subsection{Cell Positioning}

The human blood has approximately $5 \times 10^{6}$ red blood cells per $\mathrm{mm}^{3}$ [dG95]. This makes the construction of the entire geometrical layout of the cells beforehand impractical. For this reason, in the CLBlood model, a cell is probabilistically positioned in the blood volume to enable the simulation of its interactions with light. After the simulation is performed, the cell is discarded, and the process is repeated for the next cell (Figure 4). Hence, using this instantiation strategy, previously employed in the simulation of light transport in sand [KB07], we can explicitly account for cell-light interactions without having to store them. 


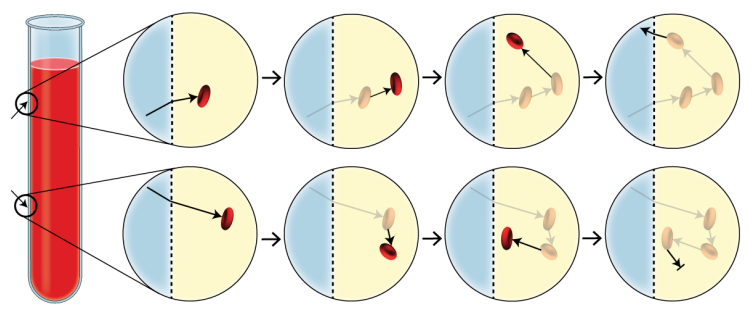

Figure 4: Sketch illustrating possible paths that can be followed by a ray traversing a blood sample in which the cells are probabilistically positioned on the fly during the simulations, and then discarded. Top: light returns to the surrounding medium. Bottom: light is absorbed.

When a ray is traversing through plasma, we first probabilistically determine the distance $d$ that the ray travels before hitting a cell. Its computation takes into account the distribution and the volume occupied by cells in the medium as well as their orientation. This is essential for the convergence of the model predictions to the same results that would have been obtained if the entire layout of the cells were geometrically assembled. Accordingly, the distance $d$ is computed using the following expression [KB07]:

$$
d=-\frac{1}{K(\vec{\omega})} \log \xi_{7}
$$

where $K(\vec{\omega})$ represents the cross-sectional area of the RBCs per unit volume given a ray traveling in a direction $\vec{\omega}$. This parameter can be further separated into two components depicted in the following integral over all possible orientations of the RBCs in the blood volume:

$$
K(\vec{\omega})=\int_{\vec{u}} n(\vec{u}) G(\vec{u}, \vec{\omega}) \mathrm{d} \vec{u},
$$

where $n(\vec{u})$ represents the number of RBCs per unit volume with the orientation given by $\vec{u}$, and $G(\vec{u}, \vec{\omega})$ corresponds to the cross-sectional area of an RBC with an orientation $\vec{u}$ and exposed to the direction $\vec{\omega}$ of the current ray. If the cells are assumed to be randomly oriented, i.e., all possible orientations have equal probability, then the parameter $n(\vec{u})$ is reduced to $H C T /(4 \pi M V C)$, where $M V C$ represents the mean volume of the RBCs. For notation simplicity, we represent the ratio $H C T / M V C$ by $\delta$ in the remainder of this section.

The formula for $G(\vec{u}, \vec{\omega})$ depends on the shape of the cell being used. Considering the NURBS representation used for our in silico cells, $G(\vec{u}, \vec{\omega})$ is given by:

$$
G(\vec{u}, \vec{\omega})=\frac{1}{2}\left(\gamma_{1}(1-|\cos \psi|)+\gamma_{2}|\cos \psi|\right),
$$

where $\gamma_{1}$ and $\gamma_{2}$ are the cross-sectional areas of the in silico cells viewed from the side and the top respectively, and $\psi$ corresponds the angle between the direction of the ray, $\vec{\omega}$, and the direction that the top of the cell is facing. For the sake of conciseness, derivation details are provided else- where $\left[\mathrm{YBK}^{*} 11\right]$. Hence, in the case in which all possible cell orientations have an equal probability, $K(\vec{\omega})$ can be analytically expressed as:

$$
K_{\text {random }}=0.5\left(\gamma_{1}+\gamma_{2}\right) \delta .
$$

Recall that during steady flow the cells are likely to have a rolling distribution (Section 3). We remark that $G(\vec{u}, \vec{\omega})$ is given by Equation 6 . Since in this case the cells are rotating with respect to an axis defined by a vector $\vec{a}$, we have $n(\vec{u})=\delta / 2 \pi$, which gives:

$$
\begin{aligned}
K_{\text {rolling }}(\vec{\omega})= & \delta \gamma_{1}(1-|\sin \beta|)+ \\
& \left.\frac{\delta}{2 \pi}\left(\gamma_{1}(2 \pi-4)+4 \gamma_{2}\right)|\sin \beta|\right),
\end{aligned}
$$

where $\beta$ is the angle between $\vec{\omega}$ and $\vec{a}$.

Under high shear rate conditions, the cells are likely to be horizontally aligned with the flow (Section 3). In this case, the calculation of the cross-sectional area per unit volume becomes simpler. We assume that that a horizontally aligned cell has its major axis on the X-Y plane. Also, since all the cells are oriented the same way, we have $n(\vec{u}, \vec{\omega})=\delta$, which gives:

$$
K_{\text {aligned }}(\vec{\omega})=\delta\left(\gamma_{1}(1-|\cos \psi|)+\gamma_{2}|\cos \psi|\right) .
$$

Once the distance $d$ is computed, the orientation of the cell is selected by sampling the probability distribution functions (PDFs) associated with the orientations that were considered while generating the distance. The PDFs for the three orientation cases were derived [YBK $\left.{ }^{*} 11\right]$ such that orientations that have a larger $G(\vec{u}, \vec{\omega})$ have a higher probability of being selected. In the random case, the PDF is given by:

$$
p_{\text {random }}(\psi, \phi)=\left(\frac{\gamma_{1}(1-|\cos \psi|)+\gamma_{2}|\cos \psi|}{\gamma_{1}+\gamma_{2}}, \frac{1}{2 \pi}\right),
$$

where $\psi$ and $\phi$ correspond respectively to the polar and azimuthal angles with respect to the ray direction $\vec{\omega}$. In the case where the cells are rolling around an axis $\vec{a}$, we know that the orientation is mostly fixed except for determining how much the cell is rotated with respect to $\vec{a}$. The PDF in this case is then given by:

$$
p_{\text {rolling }}(\theta)=\frac{\chi+\left(\gamma_{1}(1-|\cos \theta|)+\gamma_{2}|\cos \theta|\right)|\sin \beta|}{2 \pi \chi+|\sin \beta|\left[\gamma_{1}(2 \pi-4)+4 \gamma_{2}\right]},
$$

where $\theta$ is the rotation angle and $\chi=\gamma_{1}(1-|\sin \beta|)$. In the case where all cells are aligned, they all must have the same orientation $\vec{U}$. Hence, if $\vec{u}=\vec{U}$, then the PDF is given by $p_{\text {aligned }}(\vec{u})=1$. Otherwise, it is given by $p_{\text {aligned }}(\vec{u})=0$.

Finally, we randomly pick the hitting point on the cell surface among the set of points facing the ray. After the position, orientation and hitting point are selected, the model simulates the cell-light interactions as described next. 


\subsection{Cell-Light Interactions}

When the light hits a cell, we initially determine whether it is reflected or enters the cell by performing a Fresnel test using the refractive index differences between plasma and the hemoglobin solution within the cell. If the light enters the cell, we test whether it is absorbed by generating a random number $\xi_{7}$ and comparing with the probability of light absorption within the cell given by $P_{\mu_{a_{s}}}\left(\lambda\right.$, ), where $\mu_{a_{s}}$ represents the effective absorption coefficient of the solution inside the cell. If $\xi_{7}<P_{\mu_{s}}(\lambda)$, then the ray is terminated. Otherwise it is scattered.

Although the absorption probability can be computed on the fly, performance improvements are obtained by precomputing it offline since the same operations are repeated for all cells. In this case, the $P_{\mu_{a_{s}}}(\lambda)$ values are stored in a table indexed by wavelength and the incidence geometry of the incident light, i.e., the intersection point $x$ and the angle of incidence $\theta_{i}$ with respect to the cell's normal vector at this point. Due to the overall symmetry of the RBCs, only a small number of points need to be taken into account in order to obtain asymptotically convergent results in the simulations. For example, the results provided in Section 6 were obtained considering seven points and an angular resolution of $2^{\circ}$.

The probability of absorption $P_{\mu_{a_{s}}}(\lambda)$ is computed by simulating the interactions of light inside the cell. Once a ray penetrates the cell, we compute its intersection point with the internal surface of the in silico cell using a practical rayNURBS intersection algorithm [MCFS00]. At each intersection point, a Fresnel test is performed to determine whether the ray exits the cells or continues to bounce inside it. The termination of a ray due to light absorption between each bounce is also probabilistically determined using the BeerLambert law [BK10]:

$$
P_{\mu_{a_{s}}}^{r}(\lambda)=1-e^{-\mu_{a_{s}}(\lambda) d_{c}},
$$

where $d_{c}$ corresponds to the distance traveled by the ray within the cell, i.e., between bounces. The effective absorption coefficient of the solution inside the the cell can be expressed as:

$$
\begin{aligned}
\mu_{a_{s}}(\lambda)= & c_{f}\left(\left(\mathrm{SaO}_{2}\right) \varepsilon_{o h}(\lambda)+\left(1-\mathrm{SaO}_{2}\right) \varepsilon_{d h}(\lambda)\right)+ \\
& c_{d}\left(\varepsilon_{m h}(\lambda)+\varepsilon_{c h}(\lambda)+\varepsilon_{s h}(\lambda)\right)+\alpha(\lambda),
\end{aligned}
$$

where $c_{f}$ and $c_{d}$ represent the concentration of the functional and dysfunctional hemoglobins; $\varepsilon_{o h}(\lambda), \varepsilon_{d h}(\lambda), \varepsilon_{m h}(\lambda)$, $\varepsilon_{c h}(\lambda)$ and $\varepsilon_{s h}(\lambda)$ correspond to the extinction coefficients of oxyhemoglobin, deoxyhemoglobin, methemoglobin, carboxyhemoglobin and sulfhemoglobin, respectively; and $\alpha(\lambda)$ is the absorption coefficient of water. We remark that $\mathrm{SaO}_{2}$ corresponds to the oxygen saturation level. After $P_{\mu_{a_{s}}}^{r}(\lambda)$ is computed, the absorption testing is performed, i.e., if $\xi_{8}<P_{\mu_{a_{s}}}^{r}(\lambda)$, then the ray is terminated. For the offline pre-computations mentioned above, this process is repeated for $m$ rays. For example, the simulation results presented in Section 6 were obtained using $m=10^{6}$.
If the ray is scattered by the cell, its direction of propagation is perturbed using a data driven approach based on measurements of angular scattering distributions performed for single RBCs [KKKM11]. Accordingly, the polar perturbation angle $\theta_{s}$ is randomly selected from an exponential scattering distribution with a mean of $5^{\circ}$ [Lat84], while the azimuthal perturbation angle $\phi_{s}$ is randomly selected assuming an azimuthal symmetry, i.e., $\phi_{s}=2 \pi \xi_{9}$.

\section{Evaluation Framework}

The predictions provided by the CLBlood model were quantitatively and qualitatively evaluated through comparisons of modeled data with actual measured data [HYS01, MGF* 05,YYGS99] and experimental observations provided in the biomedical literature [KESJ51,Bit86,LÖ93,LHGC99, $\mathrm{SSH}^{*} 08$, Pit86]. In this section, we describe the steps taken so that the actual measurements conditions were reproduced as faithfully as possible in our in silico experiments. We also provide the values assigned for user specified parameters and the sources for the "fixed" biophysical quantities that are not normally subject to change in the simulations.

The actual measured data employed in our quantitative comparisons was obtained by placing fully oxygenated blood samples $\left(\mathrm{SaO}_{2}=1.0\right)$ inside fused quartz cuvettes. In the case of the measured spectral reflectance data, Meinke et al. [MGF*05] employed a integrating sphere spectrophotometer and placed the cuvette at an angle of $8^{\circ}$ to the incident light to exclude the Fresnel reflectance of the cuvette and measure only the diffuse directional-hemispherical reflectance. In the case of the measured scattering distribution data, Yarolavsky et al. [YYGS99] and Hammer et al., [HYS01] employed a goniophotometer and placed the cuvette at an angle of $0^{\circ}$ to the incident light. The measurements of the intensity distribution of the scattered light were taken in the range from $2.5^{\circ}-175^{\circ}$ and reported as normalized intensity values in arbitrary units (a.u.). The corresponding modeled data was obtained employing the same experimental set-ups used in the actual measurements, which were reproduced using a virtual spectrophotometer and a virtual goniophotometer [BK10]. The modeled spectral curves used in our qualitative comparisons were also obtained considering a directional-hemispherical geometry and an angle of incidence of $8^{\circ}$. It is worth noting that the scattering intensity measurements performed by Hammer $e t$ $a l$. were normalized above one, and the measurements performed by Yarolavsky et al. do not include values between $80^{\circ}$ and $100^{\circ}$. These aspects were also taken into account in the plotting of the modeled and measured scattering profiles.

In the actual experiments performed by Meinke et al. $\left[\mathrm{MGF}^{*} 05\right]$ a $0.116 \mathrm{~mm}$ thick cuvette was employed. They also measured the MVC of the RBCs in their blood samples, which was reported to be $83 \mu \mathrm{m}^{3}$. Unless otherwise stated, the same values were used in our in silico experiments, along with a cell diameter of $8.21 \mu m$ [YPR ${ }^{*} 02$ ] 
and a cell hemoglobin content equal to $29.5 \mathrm{pg}$ [LBB06]. A $2 \%$ hemolysis was observed in the actual reflectance measurements by Meinke et al. $\left[\mathrm{MGF}^{*} 05\right]$ and incorporated in the computation of the corresponding modeled reflectance data used in our quantitative comparisons. For the fixed spectral quantities used in our simulations, namely the molar extinction coefficients of functional and dysfunctional hemoglobins, the specific absorption coefficient of water, the effective absorption coefficient of plasma as well as the refractive indices of hemoglobin and fused quartz, we employed spectral curves available in the literature that have been gathered in an accompany technical report $\left[\mathrm{YBK}^{*} 11\right]$. The curve for the plasma refractive index [YBK $\left.{ }^{*} 11\right]$ used in our experiments corresponds to a $91 \%$ solution in which the concentrations of albumin, globulin and fibrinogen are $4.6 \mathrm{~g} / \mathrm{L}, 2.6 \mathrm{~g} / \mathrm{L}$ and $0.38 \mathrm{~g} / \mathrm{dL}$ respectively [MWM11]. Average values for the refractive indices of albumin, globulin and fibrinogen were also obtained using data available in the literature, 1.514, 1.511 and 1.563 , respectively [YBK $\left.{ }^{*} 11\right]$, as well as values for their molecular weights and molecular radii, which correspond to $1.13 \times 10^{-19} \mathrm{~g} /$ molecule, $2.7 \mathrm{~nm} ; 1.62 \times 10^{-19} \mathrm{~g} /$ molecule, $2.95 \mathrm{~nm}$; and $5.65 \times 10^{-19} \mathrm{~g} /$ molecule, $10.8 \mathrm{~nm}$, respectively [YBK*11]. For experiments involving hemolysis, the effective absorption coefficient of plasma was adjusted to take into account light absorption by hemoglobin. Since the presence of dysfunctional hemoglobins was not reported in the actual experiments, they were not included in the corresponding simulations either.

The rheological states in which the actual experiments were performed were also taken into account in our simulations. Unless otherwise stated, for experiments performed in a stationary (suspension) state, the RBCs were assumed to be randomly oriented (Section 3). Since the experiments by Meinke et al. [MGF* 05$]$ were performed under steady flow and high shear rate conditions, we considered the RBCs to be either randomly oriented with angular rotation $(30 \%)$ or aligned with the flow $(70 \%)$ in the corresponding simulations. These figures were selected considering experimental work [LÖ93] performed under similar rheological conditions. Since the actual measured reference data used in the quantitative comparisons was obtained either for samples with HCT below 0.4 or in a stationary (suspension) state, cell elongation was not considered in those comparisons.

\section{Results and Discussion}

Figure 5 presents comparisons of modeled reflectance curves with actual measured curves provided by Meinke et al. [MGF*05] for different HCT values. These comparisons show that the shapes of the modeled curves closely agree with the shapes of their measured counterparts. As demonstrated in the latter, below $600 \mathrm{~nm}$, a region where absorption prevails over scattering [MGF*05], the reflectance of blood increases with decreasing HCT, while above $600 \mathrm{~nm}$,
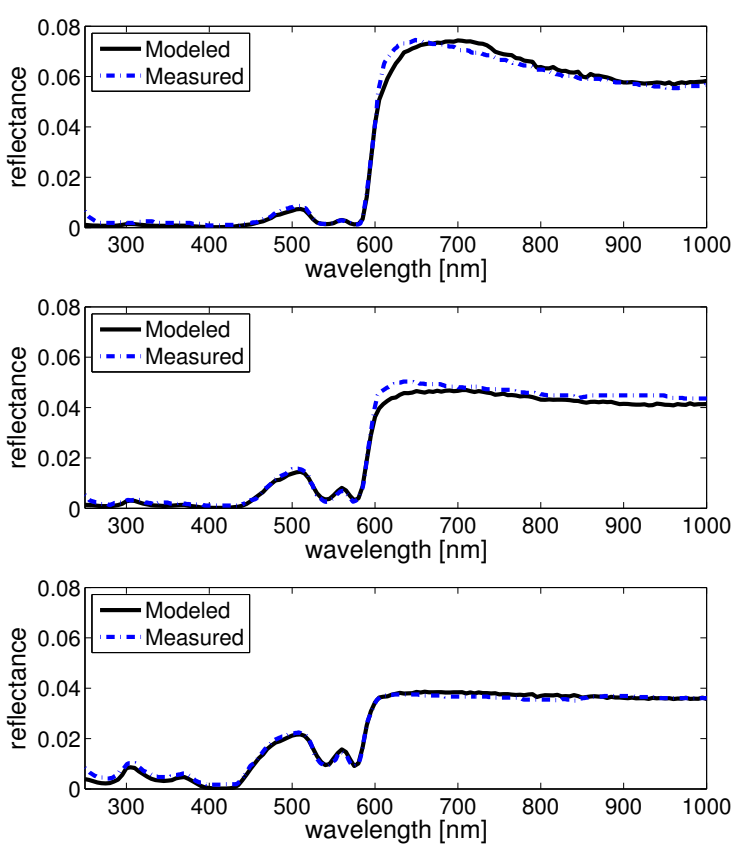

Figure 5: Comparison of modeled reflectance curves with curves measured by Meinke et al. [MGF* 05] under steady flow conditions. Top: $H C T=0.33$. Center: $H C T=0.17$. Bottom: $H C T=0.084$.
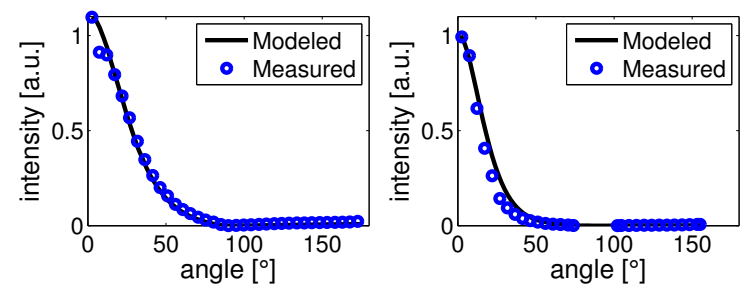

Figure 6: Comparison of modeled scattering intensity profiles with actual scattering intensity profiles measured at $514 \mathrm{~nm}$ by Hammer et al. [HYSO1] and at $633 \mathrm{~nm}$ by Yarolavsky et al. [YYGS99] for blood suspensions with $H C T=0.4$ and $H C T=0.38$, respectively. Left: $514 \mathrm{~nm}$. Right: $633 \mathrm{~nm}$.

it decreases. As it can be observed in Figure 5, the modeled curves can correctly reproduce this behavior. The comparisons depicted in Figure 5 also indicate a close quantitative agreement between the modeled and measured curves, especially considering that certain biochemical and biophysical input parameters, such as hemoglobin content and cell diameter, correspond to averaged data.

Figure 6 presents comparisons of scattering intensity profiles provided by the CLBlood model with actual scattering intensity profiles measured by Hammer et al. [HYS01] and 


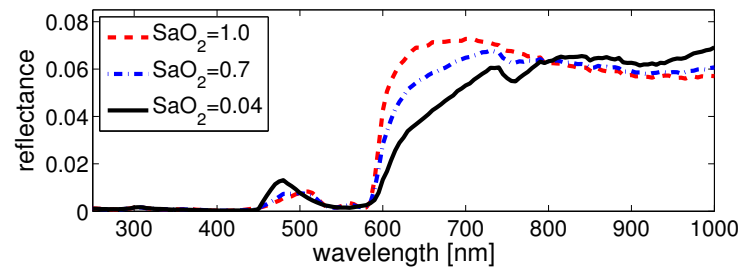

Figure 7: Comparison of modeled reflectance curves considering different levels of oxygenation $\left(\mathrm{SaO}_{2}\right)$ in whole blood suspensions with $H C T=0.4$.

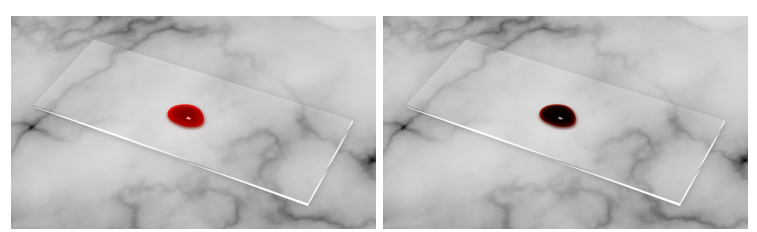

Figure 8: Renderings of blood droplets $(H C T=0.4)$ on a microscope slide showing spectral variations due to different oxygenation levels. Left: oxygenated (arterial) blood. Right: deoxygenated (venous) blood.

Yarolavsky et al. [YYGS99] below and above 600 $\mathrm{nm}$ respectively. These comparisons reveal a close quantitative and qualitative agreement between the model predictions and the measured data, demonstrating that the strong forward scattering behavior of whole blood is captured by the proposed model.

The two functional hemoglobin forms, oxyhemoglobin and deoxyhemoglobin, have the same molar extinction coefficient around $800 \mathrm{~nm}$, their isosbestic point [YPR $\left.{ }^{*} 02\right]$. By reducing the level of oxygenation, the reflectance decreases for wavelengths below this point, and increases for wavelengths above it as demonstrated in experiments by Meinke et al. [MGF* 05]. Experiments by Serebrennikova et al. [SSH* $\mathrm{SS}^{*}$ also show that a dramatic reduction of the level of oxygenation results not only in the flattening of the characteristic oxyhemoglobin "w" signature between $500 \mathrm{~nm}$ to $600 \mathrm{~nm}$, but also in a significant enhancement in the reflectance peak around $480 \mathrm{~nm}$ and in the reflectance dip around $760 \mathrm{~nm}$. The curves presented in Figure 7 indicate that the CLBlood model captures this non-trivial behavior of the functional hemoglobins, which provides the basis for noninvasive optical monitoring procedures such as pulse oximetry. This behavior is also responsible by the distinct bright red appearance of oxygenated (arterial) blood in comparison with the dark red appearance of deoxygenated (venous) blood [Fle95], which are illustrated in the images presented in Figure 8.

It has been demonstrated that the optical properties of whole blood differ markedly from those of hemoglobin solutions due to sieve and detour effects [KESJ51, Pit86, LHGC99]. The former corresponds to light traversing whole blood without encountering the RBCs, while the latter corresponds to the refraction of light between and within the RBCs. In whole blood with low hematocrit, sieve effects reduce the absorption of light by intracellular hemoglobin notably in bands of absorption maxima [Pit86, RFD*99]. For samples with physiological hematocrit $(>0.37)$, the detour effects dominate [LHGC99]. This results in an increase in the amount of light absorbed by whole blood, notably in bands of absorption minima [KESJ51,BK10], in comparison with the amount of light absorbed by solutions in which the same concentration of hemoglobin is uniformly dispersed (e.g., completely hemolysed blood samples) [LHGC99]. The curves presented in Figure 9 demonstrate that the cell-based design of the CLBlood model correctly accounts for these phenomena responsible for the distinct appearances of whole blood (intact cells) and hemoglobin solutions, which are illustrated in the images presented in Figure 10.
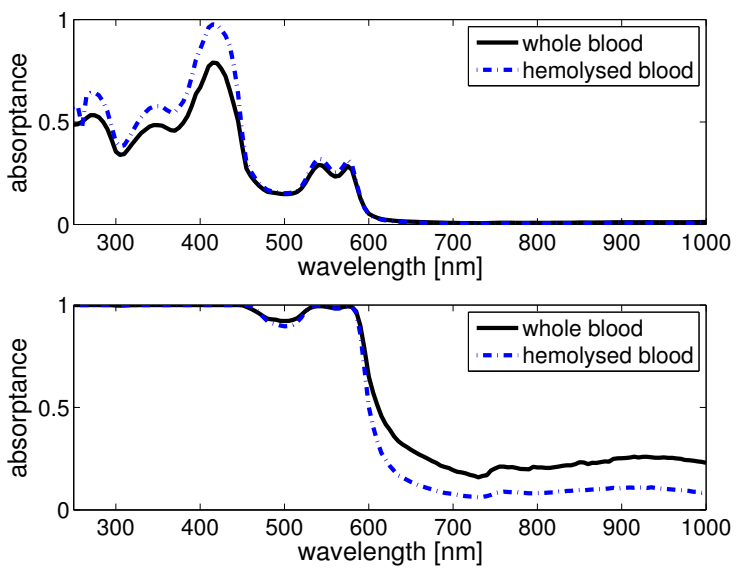

Figure 9: Comparison of modeled absorptance curves considering fully hemolysed and whole blood suspensions $\left(\mathrm{SaO}_{2}=0.7\right)$. Top: $\mathrm{HCT}=0.05$. Bottom: $H C T=0.4$.

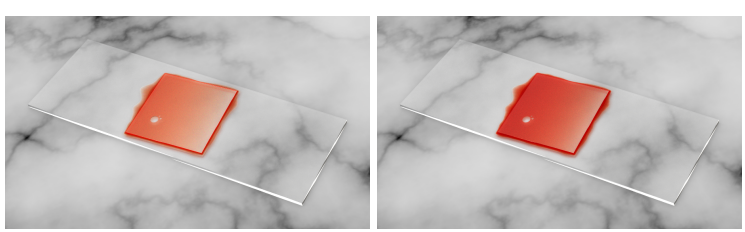

Figure 10: Renderings of blood samples $(H C T=0.4$ and $\left.\mathrm{SaO}_{2}=0.7\right)$ under a microscope slide chip illustrating the appearance differences between fully hemolysed and whole blood. Left: hemolysed blood. Right: whole blood.

Recall that motion may affect the properties of red blood cells, and consequently how light is reflect and transmitted by flowing blood. Experiments described in the literature [Bit86, LÖ93] show that as the shear rate increases, 
the RBCs start to align with their major axis parallel to the flow direction. This change of orientation, in turn, increases light reflection and reduces light transmission. We have performed simulations to examine the predictive capabilities of the CLBlood model with respect to these effects. As can be observed in Figure 11, the results of our in silico experiments are consistent with the reflection and transmission changes observed in the experiments reported in the literature.
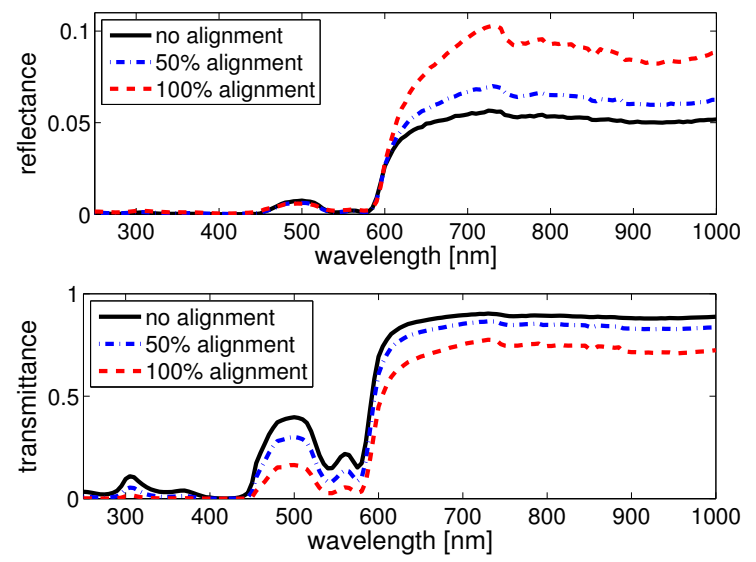

Figure 11: Comparison of modeled spectral curves considering different levels of RBC alignment for a blood sample ( $\mathrm{HCT}=0.4$ and $\left.\mathrm{SaO}_{2}=0.7\right)$ under steady flow conditions. Top: reflectance. Bottom: transmittance.

Finally, Figures 1 and 12 present rendered images to further illustrate appearance variations associated with different physiological parameters. From a visual perspective, $H C T$ and $\mathrm{SaO}_{2}$ are the most important model parameters considering samples with the same thickness and under the same rheological conditions. Note that variations on thickness may also result in noticeable (visual) appearance changes as depicted in Figure $1\left(\mathrm{HCT}=0.4\right.$ and $\left.\mathrm{SaO}_{2}=0.7\right)$. The rendering of the images $(1536 \times 1152$ pixels $)$ presented in Figures 1 and 12 took $\approx 4.5 h$, while the rendering of the remaining smaller images $(768 \times 576$ pixels, before cropping) took $\approx 2 h$ on a dual 6 core Intel Xeon X5650 processors $(2.66 \mathrm{GHz})$ with $24 \mathrm{~GB}$ RAM.

\section{Conclusion and Future Work}

We have described the first predictive model of light interaction with human blood presented in the computer graphics literature. The CLBlood model provides spectral and scattering responses for blood samples under a wide range of physiological and rheological conditions. The model predictions have been evaluated through in silico experiments, and the results of these experiments show a close quantitative and qualitative agreement with actual measured data and experimental observations reported in the biomedical literature.

Despite the comprehensive biophysically-based approach

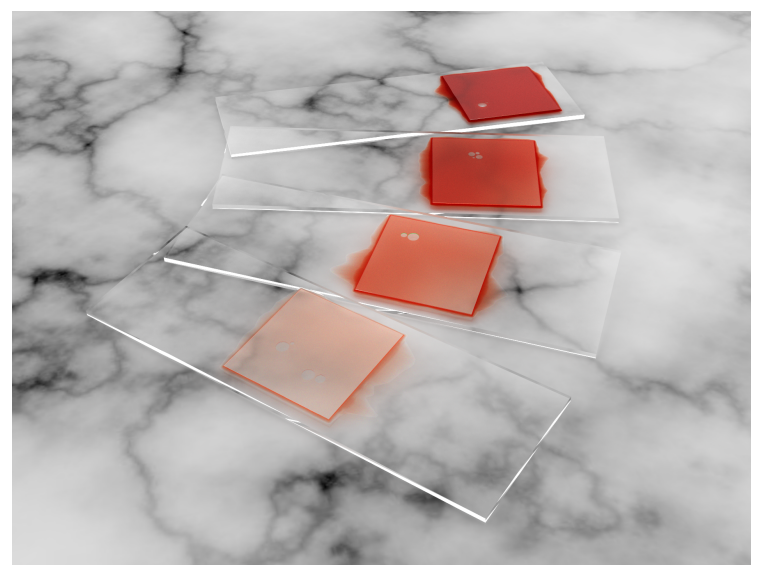

Figure 12: Rendering of blood samples showing chromatic and translucency changes caused by different HCT values. From front to back: $0.05,0.15,0.35$ and 0.49 .

employed in the design of the CLBlood model, only a small set of physiologically meaningful parameters, notably the hematocrit and the oxygen saturation level, are required to predictively control the rendering of blood samples with distinct appearances. We remark that most of the biophysical quantities employed in the simulations, such as refractive indices and absorption coefficients, are not normally subject to change, and, therefore, can be kept fixed during rendering.

Due to their stochastic nature, the simulations performed by the CLBlood model are time consuming. However, alternatives exist to enhance their accuracy to cost ratio. For example, several stages of the simulation pipeline can be effectively executed offline, and their results stored and directly accessed during rendering time.

In our future work, we intend to explore several open avenues for interdisciplinary theoretical and applied research involving the diagnosis of medical conditions through blood spectral signatures. For example, several diseases related to the abnormal occurrence of dysfunctional hemoglobins can change the color of blood and blood-perfused tissues dramatically. We also intend to investigate the effects of extreme flow and abnormal physiological conditions on the cellular anatomy, and, consequently, on the appearance of whole blood and blood-perfused tissues.

\section{References}

[AS67] ANDERSON N., SEKELJ P.: Light-absorbing and scattering properties of nonhaemolysed blood. Phys. Med. Biol. 12, 2 (1967), 173-184. 2

[Bit86] Bitbol M.: Red blood cell orientation in orbit $\mathrm{C}=0$. Biophys. J. 49 (1986), 1055-1068. 2, 3, 6, 8

[BK10] Baranoski G., Krishnaswamy A.: Light and Skin Interactions: Simulations for Computer Graphics Applications. 
Morgan Kaufmann, Elsevier, Burlington, MA, USA, 2010. 1, 2, $4,6,8$

[dG95] DE GraAfF K. V.: Human Anatomy, 4th ed. Wm. C. Brown Publishers, Dubuque, IO, USA, 1995. 2, 3, 4

[DJ06] DONNER C., JENSEN H.: A spectral BSSRDF for shading human skin. In 17th Eurographics Workshop on Rendering (2006), pp. 409-418. 2

[DRS08] Dorsey J., Rushmeier H., Sillion F.: Digital Modeling of Material Appearance. Morgan Kaufmann/Elsevier, Burlington, MA, USA, 2008. 1

[FCJ07] Frisvad J., Christensen N., Jensen H.: Computing the scattering properties of participating media using lorenz-mie theory. ACM T. Graphic 26, 3 (2007), 60:1-60:10. 2

[Fle95] Flewelling R.: Noninvasive optical monitoring. In The Biomedical Engineering Handbook (Boca Raton, FL, USA, 1995), Bronzino J., (Ed.), IEEE Press, pp. 1346-1356. Chapter 88. $2,3,8$

[FRMM06] Friebel M., Rogan A., Müller G., Meinke M.: Determination of optical properties of human blood in the spectral range 250 to $1100 \mathrm{~nm}$ using Monte Carlo simulations with hematocrit-dependent effective scattering phase functions. J. Biomed. Opt. 45, 12 (2006), 2838-2842. 1, 2, 3

[GSAA08] Gutierrez D., SERon F., A.Munoz, Anson O. Viasualizing underwater ocen optics. Comput. Graph. Forum, 2 (2008), 547-556. 2

[HTP*08] Hentschel B., Tedjo I., Probst M., Wolter M., Behr M., Bischof C., Kuhlen T.: Interactive blood damage analysis for ventricular assist devices. IEEE T. Vis. Comput. Gr. 14, 6 (2008), 1515-1522. 2

[HYS01] Hammer M., YARoslavsky A., SChWEITZER D.: A scattering phase function for blood with physiological haematocrit. Phys. Med. Biol. 46 (2001), N65-N69. 6, 7

[JAM*10] JAKOB W., ARbreE A., Moon J., BAla K., MARSCHNER S.: A radiative transfer framework for rendering materials with anisotropic structure. ACM T. Graphic 29, 4 (2010), 53:1-53:13. 2

[KB07] KimMEL B., BARANOSKI G.: A novel approach for simulating light interaction with particulate materials: application to the modeling of sand spectral properties. Opt. Express 15, 15 (2007), 9755-9777. 2, 4, 5

[KESJ51] Kramer K., Elam J., Saxton G., Jr. W. E.: Influence of oxygen saturation, concentration and optical depth upon the red and near-infrared light transmittance of whole blood. Am. J. Physiology 165 (1951), 229-246. 2, 3, 6, 8

[KKKM11] KinNUNEN M., KAUPPIla A., KARMENYAN A., MYLLYLÄ R.: Effect of the size and shape of a red blood cell on elastic light scattering properties. Biomed. Opt. Express 2, 7 (2011), 1803-1814. 2, 3, 6

[Lat84] LATIMER P.: A wave-optics effect which enhances light absorption by chlorophyll in vivo. Appl Spectrosc. 40, 2 (1984), 193-199. 3, 6

[LBB06] Lewis S., BAIn B., BATES I.: Dacie and Lewis Practical Hematology, 10th ed. Churchill Livinstone Elsevier, Philadelphia, PA, USA, 2006. 6

[LHGC99] Lovell A. T., Hebden J. C., Goldstone J. C., Cope M.: Determination of the transport scattering coefficient of red blood cells [3597-121]. In Society of Photo-Optical Instrumentation Engineers (SPIE) Conference Series (1999), Chance B., Alfano R. R., Tromberg B. J., (Eds.), vol. 3597, p. 175. 6, 8

[LÖ93] LINDBERG L., ÖBERG P.: Optical properties of blood in motion. Opt. Eng. 32, 2 (1993), 253-257. 1, 2, 3, 6, 7, 8
[McC76] MCCARTNEY E.: Optics of the Atmosphere: Scattering by Molecules and Particles. John Wiley \& Sons Inc., 1976. 4

[MCFS00] Martin W., Cohen E., Fish R., Shirley P.: Practical ray tracing of trimmed NURBS surface. J. Graph, gpu and games tools 5, 1 (2000), 27-52. 4, 6

[MGF*05] Meinke M., Gersonde I., Friebel M., HelfMANN J., MÜller G.: Chemometric determination of blood parameters using visible-near-infrared spectra. Appl. Spectroscp. 59,6 (2005), 826-835. 3, 6, 7, 8

[MMFH07] MeinKe M., ML̈ler G., Friebel M., HelfmanN J.: Optical properties of platelets and blood plasma and their influence on the optical behavior of whole blood in the visible to near infrared wavelength range. J. Biomed. Opt. 12, 1 (2007), 014024-1-014024-9. 3

[MWM11] Marieb E., Wilhelm P., Mallatt J.: Human Anatomy, 6th ed. Pearson Benjamin Cummings, San Francisco, CA, USA, 2011. 7

[OS11] Oshima S., SANKaI Y.: Development of red blood cellphoton simulator for optical propagation analysis in blood using Monte Carlo method. IEEE T. Inf. Technol. B. 15, 3 (2011), 356363. 2

[PH07] Petersch B., Honigmann D.: Blood flow in its context: Combining 3d b-mode and color doppler ultrasonic data. IEEE T. Vis. Comput. Gr. 13, 4 (2007), 748-757. 2

[Pit86] PitTMAN R. N.: In vivo photometric analysis of hemoglobin. Ann Biomed. Eng. 14, 2 (1986), 1416-1432. 6 ,

[RFD*99] Rogan A., Friebel M., Dörschel K., Hahn A., MÜLlER G.: Optical properties of circulating human blood in the wavelength range 400-2500nm. J. Biomed. Opt. 4, 1 (1999), 36-46. 2, 3, 8

[SL98] SARDAR D., LEVY L.: Optical properties of whole blood. Laser Med. Sci. 13 (1998), 106-111. 2

[SS88] Steinke J., Shepherd A. P.: Diffusion model of the optical absorbance of whole blood. J. Opt. Soc. Am. A. 5, 6 (1988), 813-822. 2

[SSH*08] Serebrennikova Y. M., Smith J. M., Huffman D. E., Leparc G. F., García-Rubio L. H.: Quantitative interpretations of visible-nir reflectance spectra of blood. Opt. Express 16, 22 (2008), 18215-18229. 2, 6, 8

[SZC73] SKALAK R., ZARDA A. T. R., CHIEn S.: Strain energy function of red blood cell membranes. Biophysical Journal 13 (1973), 245-264. 4

[TMEG85] Tycko D., Metz M., Epstein E., Grinbaum A.: Flow-cytometric light scattering measurement of red blood cell volume and hemoglobin concentration. Appl. Optics 24, 9 (1985), 1355-1365. 3

[YBK*11] Yim D., Baranoski G., Kimmel B., Chen T., MIRANDA E.: On the modeling of human blood appearance. Tech. Rep. CS-2011-30, School of Computer Science, University of Waterloo, December 2011. 2, 4, 5, 7

[YPR*02] Yaroslavsky A. N., PRIEZZheV A. V., RoDRIQUeZ J., YAROSLAVSKY I. V., BATTARBEe H.: Optics of blood. In Handbook of Optical Biomedical Diagnostics (Bellingham, 2002), Tuchin V. V., (Ed.), SPIE-Press, pp. 169-216. 2, 3, 6,7

[YYGS99] YaroslaVsKy A., YaroslaVsky I., GOLDBACH T., SCHWARZMAIER H.: Influence of the scattering phase function approximation on the optical properties of blood determined from the integrating sphere measurements. J. Biomed. Opt. 4, 47 (1999), 47-53. 2, 6, 7 